\title{
Editorial comment: the effect of neoadjuvant chemotherapy on tumour downstaging and outcomes in patients with muscle invasive bladder cancers
}

\author{
Nabil Ismaili ${ }^{1,2}$ \\ ${ }^{1}$ Faculty of Medicine, Mohammed VI University of Health Sciences, Casablanca, Morocco; ${ }^{2}$ Department of Medical Oncology, Cheikh Khalifa \\ University Hospital, Casablanca, Morocco \\ Correspondence to: Nabil Ismaili, MD. Mohammed VI University of Health Sciences, Cheikh Khalifa University Hospital, Casablanca, Morocco. \\ Email: nismaili@um6ss.ma. \\ Provenance and Peer Review: This article was commissioned and reviewed by the Section Editor Xiao Li, MD (Department of Urology, Jiangsu Cancer \\ Hospital \& Jiangsu Institute of Cancer Research \& Nanjing Medical University Affiliated Cancer Hospital, Nanjing, China). \\ Comment on: Paschalis A, Sheehan B, Riisnaes R, et al. Prostate-specific Membrane Antigen Heterogeneity and DNA Repair Defects in Prostate \\ Cancer. Eur Urol 2019;76:469-78.
}

Submitted Apr 01, 2020. Accepted for publication Apr 17, 2020.

doi: $10.21037 /$ tau-20-842

View this article at: http://dx.doi.org/10.21037/tau-20-842

Urothelial carcinoma of the bladder (also known as transitional cell carcinoma of the bladder) is the second most common malignancy of the mal genital tract in the word. It is the $10^{\text {th }}$ most common cancer. Based on the data of GLOBOCAN 2018, the estimated new cases were 549,000 and the estimated deaths was 200,000. Bladder cancer is more common in men than in women with the male/female ration of 4 . The incidences were $9.6 / 100,000$ and 3.2/100,000 in men and women respectively (1). It is the ninth most common cancer in Morocco according to Casablanca published in 2016. Tobacco is the strongest risk factor of bladder cancer worldwide and Schistosoma baematobium is the most common etiology in Egypt (2).

Radical surgery involving cystoprostatectomy in men and pelvectomy (cystectomy plus hysterectomy) in women, plus pelvic lymph node dissection, is the standard of care of muscle invasive bladder cancer (MIBC). However, in the last two decades, neoadjuvant chemotherapy (NAC) has been widely investigated in the management of patient with clinical stages T2-T4a MIBC (3). In fact, two randomize trial and one meta-analysis confirmed the positive impact of neoadjuvant cisplatin combination before radical cystectomy (RC) in MIBC. The Southwest Oncology Group (US intergroup) trial randomly assigned 317 patients to radical cystectomy (RC) versus 3 cycles of MVAC chemotherapy followed by RC (4). NAC significantly improved outcomes of patients MIBC. Pathologic complete response rate (pCR) in MVAC group was $38 \%$ vs. $15 \%(\mathrm{P}=0.001)$. Median overall survival (OS) was 77 vs. 46 months $(\mathrm{P}=0.06)$, in favor of NAC. In both groups (NAC and no NAC), improved survival was correlated with the occurrence of complete downstaging (CD), defined as the absence of residual disease (ypT0N0) in the cystectomy specimen. Five-year OS rate for patient with CD was $85 \%$. In fact, pCR is currently considered as a powerful surrogate marker of response to NAC. The largest neoadjuvant trial was conducted by the EORTC intergroup and enrolled 976 patients with stages T2/T3 and T4 bladder cancers. Patients were randomized to either radical treatment (surgery or radiotherapy) or NAC followed by radical treatment. Median OS and 3 -year OS were 44 months and $56 \%$ in the NAC group, as compared to 37.5 months and $50 \%$, respectively in the radical treatment group (5). In a meta-analysis involving more than 3,000 patients from 11 randomized trials, cisplatin neoadjuvant chemotherapy was associated with a positive absolute overall survival benefit of $5 \%$ at 5 -year (6).

More recently, several chemotherapy regimens have been investigated in neoadjuvant setting. Dose dense MVAC (ddMVAC) was investigated in a large transverse comparative study $(\mathrm{n}=1,113)$. Patients who received neoadjuvant ddMVAC showed a significantly higher rate of pathologic complete downstaging (called ypT0N0 or pCD) $(41.3 \%$ vs. 
24.6\%) and longer OS as compared to those treated with neoadjuvant gemcitabine plus cisplatin. In addition, there is a surrogate association of $\mathrm{pCD}$ disease status with OS (7). Neoadjuvant dose dense gemcitabine $\left(2,500 \mathrm{mg} / \mathrm{m}^{2}\right.$ on day 1) plus cisplatin (cisplatin $35 \mathrm{mg} / \mathrm{m}^{2}$ on days 1 and 2) (ddGC) delivered every 2 weeks for 6 cycles was investigated in a recent phase II trial conducted by the MSKCC group and showed pCR rate of $15 \%$ (8). The most common toxicities with ddGC were anemia (12\%; grade 3$)$ and hyperglycemia (12\%; grades 3 and 4 ).

In their article entitled "Tumor Downstaging as an Intermediate Endpoint to Assess the Activity of Neoadjuvant Systemic Therapy in Patients with MuscleInvasive Bladder Cancer", published in Cancer, Martini and colleagues from Tisch Cancer Institute evaluated the impact of NAC on tumor downstaging and outcomes in patients with MIBC (9). Overall, 189 and 2,010 patients were analyzed from tow cohorts, the Retrospective International Study of Cancers of the Urothelial Tract (RISC) and the National Cancer Database (NCDB) cohorts, respectively. Tumor downstaging was defined as pathologic stage (TNM) less than clinical stage. To analyze the impact of pDS (excluding pCR), the authors examined 3 subgroups: patients with no response to NAC, patients with pDS excluding pCR, and patients with pCR. Results showed that NAC is significantly associated with a significant increase in the rate of pDS as compared to cystectomy alone. In NAC group, pDS was achieved by $33 \%$ and $35 \%$ of the cases in RISC and NCDB, respectively. pCR was achieved by $20 \%$ and $15 \%$ of the cases in RISC and NCDB, respectively. In addition, $\mathrm{pDS}$ and $\mathrm{pCR}$ are associated with improved OS: 5 -year OS was $73 \%$ for patients achieving pDS and was $93 \%$ for those achieving pCR. Finally, good response to NAC (pDS excluding pCR or pCR) was associated with higher OS as compared to the absence of response to NAC.

The authors should be congratulated for this interesting work using a large dataset evaluating the effect of NAC on tumor downstaging and outcome in patient with MIBC. Their study further illustrates the significant impact of NAC in the management of MIBC and they demonstrate that pDS excluding pCR is associated with improved OS (9).

However, the study presents several limitations which warrant discussion:

* The retrospective nature of the study may introduce selection bias.

* No standardization of chemotherapy regimens in the tow cohorts. In addition, this study did not analyze the effect of dose and dose intensity of chemotherapy.

* The absence of centralized pathological review in this study.

* The follow-up time was not indicated.

Only a few studies have investigated the effect of NAC on tumour downstaging (downstaging excluding pCR) and outcomes and the value of pDS as marker of response to NAC in patients with stage T2-T4N0M0 MIBC (Table 1).

In a study conducted by a Swedish group (Nordic 1 and 2), including 449 patients treated by $\mathrm{RC}$, the rate of pCD was higher in the NAC arm (22.7\%) compared with the $\mathrm{RC}$ alone arm (12.5\%) $(\mathrm{P}=0.006)$. The difference was significant for $\mathrm{T} 3$ tumors (pDS was $17.6 \% v s .6 .1 \%$, $\mathrm{P}=0.014)$, but no significant for $\mathrm{c} T 2$ tumors $(\mathrm{P}=0.103)$. Fiveyear survival was $77.5 \%$ for patients with organ-confined (OC) tumors in the NAC arm as compared with $64.6 \%$ in patients with $\mathrm{OC}$ in the control arm $(\mathrm{P}=0.005)$. For patients with non-organ-confined tumors, no benefit was observed in the NAC arm (10).

The MSKCC phase II trial enrolled 49 patients with clinical stage $\mathrm{T} 2$ to $\mathrm{T} 4 \mathrm{aN} 0 \mathrm{M} 0$ disease to investigate the effect of induction ddGC chemotherapy on tumor downstaging $(<\mathrm{pT} 2)$ and outcomes. The primary end point of the study was met, the pathologic response rate and pT0 rates were $57 \%$ and $15 \%$, respectively. For patients who underwent RC, responders to chemotherapy $(<\mathrm{pT} 2 \mathrm{~N} 0)$ had significantly better RFS compared with non-responders ( $\geq$ pT2N0 disease) $(\mathrm{P}=0.004)$. The 2 -year RFS and OS rates in responders and nonresponders were $96-96 \%$, and 52-84\%, respectively $(\mathrm{P}=0.043)$ (8).

The effect of NAC on tumour downstaging was also explored in patients with node positive disease. ZargarShoshtar et al. reported a pCR and pDS rates of 14.5 and $27 \%$, respectively, in a multi-institutional study (European and American) of cN1-3 patients who were treated with induction MVAC or GC chemotherapy followed by RC. Median OS was not reached, 45 and 14 months for patients with $\leq \mathrm{p} T 1 \mathrm{~N} 0$, p $22-4 \mathrm{aN} 0$ and $\mathrm{p} T \mathrm{xN} 1-3$ disease, respectively $(\mathrm{P}<0.001)$. $\mathrm{pN} 0$ rate was $48 \%$. On multivariate analysis, OS was associated with $\mathrm{pN} 0$ and negative margins (11).

A population-based study $(n=659)$ explored the effect of NAC in patient with stage $\mathrm{cN}+\mathrm{MIBC}$. pCD was defined as $\leq(y)$ pT1N0 at RC. For $\mathrm{cN} 1$ and $\mathrm{cN} 2 / 3$ disease, $31 \%$ and $19 \%$ of the cases proved to be pN0 at upfront RC. In $\mathrm{cN} 1, \mathrm{pCD}$ was achieved in $39 \%$ following NAC versus $5 \%$ for upfront $\mathrm{RC}(\mathrm{P}<0.001)$. For $\mathrm{cN} 2 / 3 \mathrm{MIBC}$ disease, rates of pCD were $27 \%$ vs. $3 \%(\mathrm{P}<0.001)$. Three-year OS for pCD and ypCD groups were $81 \%$ and $84 \%$, respectively. 
Table 1 Trials and studies investigating the effect of NAC on tumour downstaging (pCR and downstaging) and outcomes (progression free survival and overall survival) in patients with MIBC

\begin{tabular}{|c|c|c|c|c|c|c|}
\hline Clinical study (design) & $\mathrm{N}$ & Original stage & Treatments & pDS rate & pCR rate & Effect of pDS on OS \\
\hline $\begin{array}{l}\text { RISC and NCDB } \\
\text { (retrospective) (9) }\end{array}$ & $\begin{array}{l}181 \text { (RISC) and } \\
2,010 \text { (NCDB) }\end{array}$ & T2-4NOMO & NAC & $33 \%$ and $35 \%$ & $20 \%$ and $15 \%$ & $\begin{array}{c}\text { pDS was associated with } \\
\text { better OS }\end{array}$ \\
\hline $\begin{array}{l}\text { Nordic Trials } 1 \text { and } 2 \\
\text { (retrospective) (10) }\end{array}$ & 449 & $\begin{array}{l}\text { T2-T4aNxM0, } \\
\text { pT4aNO-N+M0 }\end{array}$ & NAC vs. RC & - & $\begin{array}{c}22.7 \% \text { vs. } \\
12.5 \%\end{array}$ & $\begin{array}{l}\text { pCR was associated with } \\
\text { better OS in NAC group }\end{array}$ \\
\hline
\end{tabular}

pDS, pathologic downstaging; pCR, pathologic complete response; SWOG, Southwest Oncology Group; MVAC, Methotrexate, Vinblastine, Doxorubicin and Cisplatin; RC, radical cystectomy; dd-MAVAC, dose dense MVAC; GC, Gemcitabine Cisplatin; MSKCC, Memorial Sloan-Kettering Cancer Center; dd-GC, dese dense Gemcitabine Cisplatin; RISC, Retrospective International Study of Cancers of the Urothelial Tract; NCDB, National Cancer Database; NAC, neoadjuvant chemotherapy.

Three-year OS rates were $66 \%$ versus $37 \%$ for $\mathrm{cN} 1$ disease and $43 \%$ versus $22 \%$ for $\mathrm{cN} 2-3$ disease, in favor of NAC $(\mathrm{P}<0.001)$. In multivariate analyses, NAC was associated with pCD and a $53 \%$ decreased risk of death (HR, 0.47) (12).

In conclusion, NAC for stage T2-T4 N0/N+ M0 MIBC is associated with a clinically significant pathological response $(\mathrm{pPD} / \mathrm{pCD})$, even in $\mathrm{cN} 1-3$ disease. The best outcomes are seen for patients receiving induction ddMVAC and ddGC chemotherapies. Pathologic downstaging is associated with improved PFS and OS and should be considered as a powerful surrogate marker which can be used to assess the efficacy of new neoadjuvant treatments.

\section{Acknowledgments}

Funding: None.

\section{Footnote}

Conflicts of Interest: The author has completed the ICMJE uniform disclosure from (available at http://dx.doi. org/10.21037/tau-20-842). The author has no conflicts of interest to declare.
Ethical Statement: The author is accountable for all aspects of the work in ensuring that questions related to the accuracy or integrity of any part of the work are appropriately investigated and resolved.

Open Access Statement: This is an Open Access article distributed in accordance with the Creative Commons Attribution-NonCommercial-NoDerivs 4.0 International License (CC BY-NC-ND 4.0), which permits the noncommercial replication and distribution of the article with the strict proviso that no changes or edits are made and the original work is properly cited (including links to both the formal publication through the relevant DOI and the license). See: https://creativecommons.org/licenses/by-nc-nd/4.0/.

\section{References}

1. Bray F, Ferlay J, Soerjomataram I, et al. Global Cancer Statistics 2018: GLOBOCAN Estimates of Incidence and Mortality Worldwide for 36 Cancers in 185 Countries. CA Cancer J Clin 2018;68:394-424.

2. Ismaili N, Amzerin M, Flechon A. Chemotherapy in advanced bladder cancer: current status and future. J 
Hematol Oncol 2011;4:35.

3. Ismaili N. Neoadjuvant Chemotherapy: A New Standard for Muscle Invasive Bladder Cancer? Gulf J Oncolog 2017;1:82-5.

4. Grossman HB, Natale RB, Tangen CM, et al. Neoadjuvant chemotherapy plus cystectomy compared with cystectomy alone for locally advanced bladder cancer. N Engl J Med 2003;349:859-66. Erratum in: N Engl J Med 2003;349:1880.

5. International Collaboration of Trialists; Medical Research Council Advanced Bladder Cancer Working Party (now the National Cancer Research Institute Bladder Cancer Clinical Studies Group); European Organisation for Research and Treatment of Cancer Genito-Urinary Tract Cancer Group; Australian Bladder Cancer Study Group; National Cancer Institute of Canada Clinical Trials Group; Finnbladder; Norwegian Bladder Cancer Study Group; Club Urologico Espanol de Tratamiento Oncologico Group, Griffiths G, Hall R, Sylvester R, et al. International phase III trial assessing neoadjuvant cisplatin, methotrexate, and vinblastine chemotherapy for muscleinvasive bladder cancer: long-term results of the BA06 30894 trial. J Clin Oncol 2011;29:2171-7.

6. Advanced Bladder Cancer (ABC) Meta-analysis Collaboration. Neoadjuvant chemotherapy in invasive bladder cancer: update of a systematic review and metaanalysis of individual patient data advanced bladder cancer (ABC) meta-analysis collaboration. Eur Urol 2005;48:2025; discussion 205-6.

Cite this article as: Ismaili N. Editorial comment: the effect of neoadjuvant chemotherapy on tumour downstaging and outcomes in patients with muscle invasive bladder cancers. Transl Androl Urol 2020;9(3):977-980. doi:10.21037/tau-20-842
7. Peyton CC, Tang D, Reich RR, et al. Downstaging and Survival Outcomes Associated With Neoadjuvant Chemotherapy Regimens Among Patients Treated With Cystectomy for Muscle-Invasive Bladder Cancer. JAMA Oncol 2018;4:1535-42.

8. Iyer G, Balar AV, Milowsky MI, et al. Multicenter Prospective Phase II Trial of Neoadjuvant Dose Dense Gemcitabine Plus Cisplatin in Patients With MuscleInvasive Bladder Cancer. J Clin Oncol 2018;36:1949-56.

9. Martini A, Jia R, Ferket BS, et al. Tumor Downstaging as an Intermediate Endpoint to Assess the Activity of Neoadjuvant Systemic Therapy in Patients With MuscleInvasive Bladder Cancer. Cancer 2019;125:3155-63.

10. Rosenblatt R, Sherif A, Rintala E, et al; Nordic Urothelial Cancer Group. Pathologic downstaging is a surrogate marker for efficacy and increased survival following neoadjuvant chemotherapy and radical cystectomy for muscle-invasive urothelial bladder cancer. Eur Urol 2012;61:1229-38.

11. Zargar-Shoshtari K, Zargar H, Lotan Y, et al. A Multi Institutional Analysis of Outcomes of Patients with Clinically Node Positive Urothelial Bladder Cancer Treated with Induction Chemotherapy and Radical Cystectomy. J Urol 2016;195:53-9.

12. Hermans TJ, Fransen van de Putte EE, Horenblas S, et al. Pathological downstaging and survival after induction chemotherapy and radical cystectomy for clinically node-positive bladder cancer-Results of a nationwide population-based study. Eur J Cancer 2016;69:1-8. 